\title{
Bitcoin: an alternative currency to pay for goods and services or a useful investment tool?
}

\author{
Zuzana Rowland ${ }^{1}$, Petr Suler ${ }^{1, *}$, and Bohdana Cajkovicova ${ }^{1}$ \\ ${ }^{1}$ Vysoka skola technicka a ekonomicka v Ceskych Budejovicich, Okruzni 517/10, 37001 Ceske \\ Budejovice, Czech Republic
}

\begin{abstract}
Research background: Bitcoin is defined as digital money in a peer-to-peer decentralized payment network, an amalgam hybrid between fiat and commodity currency without a real value. This digital currency is also independent of any government or currency administration.

Purpose of the article: This article explores whether bitcoin works as a medium of exchange or relates to assets, focusing on its current use and future utility regarding its characteristics.

Methods: Analysing bitcoin statistical features, we found no connection with traditional asset categories such as stock, bonds and commodities either in intermediate time, or periods of financial crises.

Findings \& Value added: The study suggests that investors' abiding interest in bitcoins can have a positive impact on their liquidity in the real time.
\end{abstract}

Keywords: volatility; bitcoin; cryptocurrency; peer-to-peer; investment

JEL Classification: $G 10$; $G 11 ; G 14 ; G 15$.

\footnotetext{
* Corresponding author: petr.suler@,cez.cz
} 


\section{Introduction}

Bitcoin, first designed by Satoshi Nakamoto, comprises a new financial asset involving a peer-to-peer electronic cash system which enables online payments directly from one side to the other without interfering with any financial institution. Therefore, contrary to other financial assets, bitcoin has no connection with any governing body or physical representation (Kliestik et al. 2020). Its value is not dependent on any fixed assets or national economy. Rather than that, it is based on a secured algorithm monitoring all transactions. The exchange potential of bitcoin lies in its low costs, peer-to-peer system and global and governmental design. However, users may be discouraged by the obscurity of the system and insufficient acceptance of bitcoin within transaction schemes (Urquhart and Zhang, 2018).

The massive expansion of bitcoins poses a radical challenge to politicians and policymakers, as this digital currency represents money and, at the same time, opens up new horizons for enterprises. In regard to galloping inflation, bitcoins have become a popular topic widely discussed throughout the media, governing bodies and academic community. Despite the assiduous attention of the media, bitcoin has not been subject to a careful analysis on academic grounds so far, thus giving space to an extensive exploration (Lucey et al. 2018).

If bitcoin is used mainly as a currency to pay for goods and services, it will enter into keen competition with fiat currencies, such as American Dollar. This will have a deep impact on fiat currencies and monetary policies adopted by the central bank. On the contrary, if this digital money is used for worthwhile investments, it will go into fierce competition with a large number of other disposable assets, such as bonds, stock and commodities, or, alternatively, its role will be rather subordinate. The presented study aims to identify the bitcoin liquidity compared to current currencies and financial assets using intraday data. To achieve this objective, testable hypotheses were formulated as follows:

Is bitcoin a currency or asset?

Are bitcoins mainly used as an alternative currency to pay for goods and services, or are they mainly used for investments?

What is the liquidity of bitcoins?

\section{Literature research}

One of the areas of research mentioned in the professional literature is the relationship between bitcoins and other commercial assets. The question here is to decide whether to include bitcoins among diversifiers, hedging, or a safe refuge from other financial assets. Dyhrberg (2016) suggests that bitcoins can function as hedging against American Dollar and the British Stock Market, or provide hedging services similar to gold. Cryptocurrencies such as bitcoins or ethereum have attracted meticulous attention from the media, financial industry, academic workers, policy-makers and consumers. Consumers may consider cryptoassets appealing for various reasons: a medium of exchange for pseudonym transfers; prosperity resource, unless agents trust conventional monetary units, banks or central banks; assets for speculating with potential capital profits; illegal actions (compare: Houben and Snyers, 2020).

Cryptocurrencies might also be attractive to consumers interested in technologies and new applications, either for economic reasons, or other. What percentage of the population has bought crypto-assets so far? Which financial possibilities do people who have invested in crypto-assets have? Are those people aware of potential risks? What is the role of the trust in an institution or conventional money in the demand for cryptocurrencies? If so, which institutions are trusted? Which motives lead non-owners to consider accepting a cryptocurrency? Were the data from the three national-representative surveys in Austria in 2018 and 2019 used? Roughly 1.6\% of Austrians own crypto-assets and 5\% can be regarded 
as potential adepts. Owners have more thorough financial knowledge on average and are more tolerant to potential risk than non-owners; owners do not consider mistrust to banks or conventional currencies as a decisive factor. The most powerful stimuli for the ownership are an expectation of profit-making and believing that crypto-assets will have payment benefits. Most adopters or potential adopters believe in both. The high volatility or risk of fraud or online theft significantly reduces the demand for cryptocurrencies (Stix, 2021). FigáTalamanca, Focardi and Patacca (2021) focuses on examining effects of cyber-attacks of hackers and the follow-up insecurity on the bitcoin market.

Can bitcoin protect investors from harsh market turbulences? A bear market caused by Covid-19 pandemic provides an initial base for testing the traits of a safe refuge of bitcoins. The bear market Covid-19 has suffered first heavy losses on the market from the date of entering into the active negotiation with bitcoins (Conlon and McGee, 2020). This dramatic slump opens a space for timely testing frequently-discussed traits of the safe refuge of bitcoins. Is bitcoin a medium of exchange of an asset? Or, more specifically, what is its current and future most effective use regarding its features?

Bitcoin has captured rapt attention by both - media and investors. What is the reason for such concern? This increased recognition can be attributed to its innovative features, simplicity, transparency and growing popularity (Urquhart, 2016). On the other hand, bitcoin presents huge challenges and opportunities for policy-makers, economists, businessmen and consumers. Cryptocurrencies are winning users' trust by having the whole history of transactions open to the public. In return, the history of transactions keeps records of all behaviour patterns of cryptocurrency users. We use data mining to analyse and summarize the existing research on examining the knowledge of transactions in the cryptocurrency. In particular, we divide the research into three categories: transaction tracing, interconnecting blockchain addresses, analysing consumers' behaviour and studying individual consumers. The economy of cryptocurrency allows predicting various new and detrimental innovations, financial services using the blockchain system in particular (Lui et al., 2021).

Urquhart (2018) shows that according to Google Trends, the special attention devoted to bitcoins reflects the volatility and volume traded on the market in previous days. Google Trends also indicates how many times keyword ‘bitcoin' was searched via Google; therefore, this website carefully monitors the attention paid by uninformed individuals collecting more information about bitcoins. On the other hand, well-informed investors, with a vast knowledge of cryptocurrencies, are not likely to search for it through Google; they will tweet it instead. These tweets may involve coverage comments concerning bitcoin or predicting future price development of bitcoin, or just expressing a personal opinion. Contrary to previous studies, these inquiries focus on Twitter tweets, extensively used by well-informed investors, rather than on Google Trends. Shen, Urquhart and Wang (2019) found out that the amount of tweets is highly impactful to the trading volume of the following day and realized volatility, supported by linear and non-linear Granger causality tests. The presented survey contributes to the growing amount of literature on bitcoins by analysing the relation between investors' attention and bitcoin yields, trading volume and realized volatility.

Our key objective is to understand the dynamics of cryptocurrency prices, in particular to explore how the information on prices is transferred between different cryptocurrency stock markets and between cryptocurrency and traditional markets. For these purposes, Giundici and Abu-Hashish (2019) laid out an expanded autoregressive vector model to expound upon the development of bitcoin prices. The expansion is based on network models which work better than simple autoregressive models, as they introduce a current contagious component describing contagion effects between prices. The suggested model can accurately depict a correlation structure between bitcoin prices on various exchange markets. These commercial venues appear relatively strong compared to low correlations between bitcoin prices and traditional assets. The model can also more precisely predict bitcoin prices given to a simpler 
autoregressive design. A big advantage of the elaborated type is its great utility for predicting bit prices. The model can predict bitcoin prices with a deviation estimated roughly to $11 \%$ of the average price. However, this inconsistency is markedly different in various foreign exchange markets: prices of central bitcoin stock markets are much easier to predict. The involvement of the current component into the prediction model leads to higher prediction accuracy on almost all markets than a simple autoregressive design.

The bitcoin network has recently seen an inflow of investors, enterprises and corporations, at the same time extending services and product supplies. Bitcoin has thus become a major source of the decentralized cryptocurrency.

A data analysis of available and used resources revealed that a content meta-analysis and descriptive statistics of cryptocurrency behaviour appear to be the best method of finding answers to the formulated hypotheses. The results will be highly instrumental in making a decision whether to invest in cryptocurrencies.

\section{Data and methods}

Bitcoin has been jostling for our attention for over eleven years, yet often anxiously avoided. Many people consider this digital money a hyped-up or, even, fraudulent project. A lot of indepth analyses concerning the future of bitcoin have been carried out, indicating inconsistent results. Cryptocurrency behaviour is very hard to estimate and predict, and nearly impossible to control. Despite this, it is very likely that bitcoin will bring about a substantial change in our lives. In this article, we evaluate bitcoin behaviour using reliable and effective methods. To detect cryptocurrency behaviour, we apply descriptive statistics, assessing bitcoin minimums, maximums, trends and mean values. The analysed data are collected from https://coinmarketcap.com/. The content meta-analysis allows us to minutely scrutinize the methods and results we had examined when looking for answers to the hypotheses.

\section{Results}

The answer to the question whether bitcoin can serve as hedging or a safe harbour against global currencies is found in the relationship between bitcoins and currencies at the clock rate. A regressive analysis shows that bitcoin functions as an intraday hedging, diversifier and safe repository for specific currencies (Urquhart and Zhang, 2019). This study widely applies EGARCH models to explore effects of cyberattacks of hackers on the follow-up insecurity on the bitcoin market. These prototypes work with fictional variables up to five days after the incidents. Since the volatility clustering appears to be a stylized reality of financial markets, the selected model can identify potential asymmetry in the volatility of bitcoin yields. The survey also provides the analysis of volatility effects with another influential cryptocurrency market - Ethereum. Even though the principal purpose of Ethereum is significantly different from bitcoin, both cryptocurrencies usually reach high circulation figures (Borri, 2019).

The findings indicate a sharp rise in volatility. The study detected two major effects - the current effect and delayed effect. The current effect might have been brought about by an increased feeling of insecurity on the stock market affected by a hacker incident. A plausible explanation of the delayed effect may be that cyberattacks probably take place on smaller stock markets, as they are usually less secure than their larger counterparts, so the information travels more slowly (Grobys, 2021). (Zhu et al., 2021) look into relationships between new avid interest of investors and bitcoin financial characteristics, i.e. the rate of return and realized volatility, both constituting two most distinctive features of one specific asset. With the establishment of cryptocurrency stock markets in 2017, the price of bitcoin dramatically soared. VAR Model presents a linear model which makes predictions of variables from the 
previous values. This design has a great utility for estimating and predicting financial assets. The results show that the rate of return of bitcoins is strongly dependent on utterly compelling investors' attention (Zhu et al., 2021).

Figa-Talamanca, Focardi and Patacca (2021) applied the dynamic factor analysis of modelling mutual behaviour of bitcoins, ethereum, litecoin and monero as a representative basket of classes of cryptocurrency assets. Empirical results suggested that a basket price depends on two dynamic factors. More precisely, the authors detected one integrated and one stationary factor until the end of August 2019, and two integrated factors later. Based on this evidence, they defined a long-term and short-term business strategy, which turned to be profit-making when the second factor was stationary. Hudson and Urquhart (2021) analysed technical trading rules governing cryptocurrency markets using statistical figures of two bitcoin markets (CoinDesk and Bitstamp) and three other popular cryptocurrencies, revealing that the technical trading rules offer substantially higher risk-free yields than a simple strategy of purchase and possession. Investors pay attention to the technical trading rules, implementing them as a part of their investment strategies. We examine whether the market with high volatility caused by Covid-19 pandemic has gained some diversification advantages from bitcoin possession. In particular, we quantify a relative change in a portfolio value at risk (VAR) and conditional value at risk (CVAR). These are the two main criteria of earnings risk. Analysing adverse financial impacts on Portfolio S\&P 500 diversified by allocation to bitcoin, we revealed that this digital money does not provide a safe harbour. During the monitored period, S\&P 500 and bitcoin were in a precarious position, resulting in an increased earnings risk for an investor and the allocation to bitcoin (Conlon and McGee, 2020).

So what is so appealing about bitcoins? To answer this question, we explored the relationship between investors' general interest and bitcoin essentials, revealing that the realized volatility and sheer volume of bitcoins are decisive factors to attract investors' attention for the following day. The volatility and volume from the previous day and yields from the previous two days are the main criteria to command investors' attention to bitcoins. Upon dividing the collected data into two component samples, we found out that the abovementioned situation applied no sooner than in October 2013. The results thereby indicate that bitcoin appeals to investors mainly after an increase in volatility and volume of traded bitcoins (Urquhart, 2018). Is bitcoin hedging abilities the same as those of gold? We examined the hedging abilities of bitcoins by the GARCH asymmetric method, widely used for gold inspection. The results revealed that bitcoins can be effectively used as hedging against shares in the Financial Times Stock Exchange Index. Bitcoins also have a short-term utility as hedging against American Dollar. To some extent, bitcoins have the same hedging abilities as gold and can thereby be involved in sophisticated instruments used by market analysts to hedge against specific market risks. Bitcoins showed exceptional hedging abilities against American Dollar on a short-term basis, which means that high-rate trading in bitcoins creates favourable conditions for implementing this type of hedging. We may conclude that bitcoin has achieved a dominant position on the market with its outstanding ability to analyse portfolios and risk management. This digital currency can thereby provide strong hedging against FTSE Index and American Dollar. Along with gold and other assets, bitcoin can be included in the list of instruments for minimizing risks (Dyhrberg, 2016).

The prevailing trends on global financial markets indicated a sharp rise in cryptocurrencies thanks to the popularity of the blockchain technology, and its impregnable position in the area of digital finance. A dramatic surge in cryptocurrencies set economists casting doubts upon the established practices. In particular, an interaction between two different types of financial markets has recently been discussed as a burning experimental issue, discovering specific relations and differences between dominant cryptocurrencies and fiat money. The main objective was to carry out an analysis of attaching the importance to 
bitcoin to explore strong relationships and direct and indirect significant relations. In this research, bitcoin, regarded as the predominant cryptocurrency on the market, and 50 other conventional currencies were subjected to a correlation analysis, HT (Hierarchical Tree) and MST (Minimum Spanning Tree). Academic workers and economists can apply the results in further research in this field. Apart from fiat currencies, the presented survey deals with bitcoin as a major currency. Regarding large fluctuations in bitcoin prices, it was observed that bitcoin could not have been highly influential in MST analysis. However, with the increasing number of cryptocurrencies involved in this research, these digital monetary units could play a leading role within fiat currencies. A detailed inspection of these relationships may reveal substantial changes in prices and easily trace the monetary network. Considering cryptocurrencies' huge contribution to the economic environment, this virtual money would more closely correlate and interconnect with fiat currencies or, even, act as their acceptable substitute (Dyhrberg, 2016).

Bitcoin's volatility, the stock market and investors' sentiment - are these factors intertwined? Analysing bitcoin behaviour and the influence of investors' sentiment, S\&P 500 profit and VIX yields on bitcoin's volatility using GARCH and EGARCH suggest that bitcoin's volatility is the most stable within speculative periods. Bitcoin has become one of the decisive and by far the biggest cryptocurrency regarding the market funding. The submitted study comes up with new evidence on the trends in bitcoin's volatility within various periods, detecting the influence of the S\&P 500 rate of return, VIX rate of return and investors' sentiment on bitcoin's volatility. The obtained results suggest that bitcoins might offer a safe refuge. Bitcoin investors may ponder the sentiment on the stock market echoing from social networks rather than the market volatility, when devising their investment strategies. It indicates that bitcoin investors are more 'technological' and pay assiduous attention to information coming from these media. It was also proved that bitcoin's volatility behaves differently through the time. Thus, bitcoins provide a safe refuge within periods of high market volatility; however, they become a sound investment for speculating investors when stock markets achieve relative stability (Lopez-Cabarcos et al., 2021). Expanding services and product offers, the bitcoin network has recently attracted prospective investors, enterprises and corporations. This digital currency has also become the principal source of cryptocurrency. Although an extensive survey on analysing bitcoin networks was carried out, little has been done on predicting bitcoin prices.

The presented study focused on predicting bitcoin prices and its changes using the grey system theory. For this purpose, we use a first order grey model (GM (1.1)). This design applies a first order differential equation for modelling time series trends. Regarding the fact that the grey system theory enables low-data and incomplete-information predictions, we adopted this convenient method to forecast bitcoin prices for the following day. The results suggest that prognoses of bitcoin prices come true using GM (1.1) within a period of 5 days with standard deviation 1.14\% (Faghih Mohammadi Jalali and Heidari, 2020). Although an extensive survey on analysing bitcoin networks was carried out, little has been done on predicting bitcoin prices. Upon interpreting high-rate data, we measured the effects of tweets on bitcoin's liquidity in the real time. We found out that $1 \%$ growth of tweets results in approximately $7 \%$ improvement of liquidity within five or ten minutes. An accurate timeline clearly shows that a positive effect of tweets wears off roughly after one hour. The research further disclosed that impacts on the liquidity are stronger when tweets attract more attention. The presented study thereby suggests that investors' keen interest in this digital currency may dramatically improve bitcoin liquidity in the real time (Choi, 2021).

\section{Discussion}

Is bitcoin a currency or an asset? 
The demand for bitcoins was comparable with the demand for other monetary units issued by the government in 2018 , as estimated from its market funding. Only six monetary units issued by the government, of which the data are easily available, received the market funding (in bil. USD) larger that the average bitcoin market funding: American Dollar (3411.50), Russian Ruble (256.33), Hong-Kong Dollar (217.71), Brazilian Real (195.91), South-Korean Won (156.61) and Czech Crown (144.40). On its minimum, the bitcoin market funding outpaced all but 18 currencies with accessible data, which equals 83rd percentile of the money at issue. On the other hand, the maximum amount of market funding exceeded all but the American Dollar, reaching 98th percentile.

The bitcoin market funding is available on website CoinMarketCap.com (2021). To facilitate the comparison with the above-mentioned base money issued by the government, we first focus on the bitcoin market funding in 2018. As of 1st January 2018 until 31st December 2018 the bitcoin market funding ranged between the minimum 56.40 (15th December) and maximum 294.22 (6th January) bil. USD. The average market funding equaled 129.27 bil. USD, whereas the median was at 117.51 bil. USD (7th July), with standard deviation 40.54 bil. USD.

We used the bitcoin market funding financed by government money as an estimate of its specific requirements, showing that a similar demand for bitcoins is comparable with the demand for lots of government monetary units. Actually, only six out of one hundred and six currencies issued by the government, with easily available data, had been better funded than an average bitcoin market funding in 2018 . All the same, we cannot identify to which extent we deal with the transaction demand for bitcoins rather than speculations. Therefore, we cannot safely conclude whether bitcoins are broadly accepted, or will rather be negotiated in the future. However, we confirm that a routine use of bitcoins as a means of exchange has met requirements for being called money among certain transaction instruments, although only within a limited domain (Hazlett and Luther, 2020).

A specific algorithm determined 21 mil. bitcoins to go into circulation. This digital unit constitutes an autonomous decentralized currency, completely independent from traditional currencies such as crown, euro etc.

Are bitcoins used mainly as an alternative currency to pay for goods and services, or are they better applied as an investment?

An analysis of statistical features of the cryptocurrency indicated no relation to traditional types of assets, such as shares, bonds and commodities either in standard periods, or periods of financial crises. A close inspection upon bitcoin public ledgers revealed that investors possess roughly one third of bitcoins. These are mostly users who accept no other money than bitcoins, never sending them to others. Shareholders keep bitcoins for investment purposes rather than transactions, i.e. means of exchange (Baur, Hong and Lee, 2018). The submitted document touched on the issue of whether bitcoin is either a means of exchange, or (speculative) investment asset. Our research revealed that bitcoin return features are significantly different from traditional types of assets including currencies, offering huge advantages in diversifications within standard periods and periods of economic recessions. Upon examining bitcoin public ledgers, we found out that roughly one third of bitcoins is possessed by investors - users accepting no other currency but bitcoin and never sending this virtual money to others. We may reasonably conclude that a minority of consumers - both in number and bitcoin balance - use bitcoins as a means of exchange. It suggests that bitcoins are currently kept for investment purposes rather than transactions, i.e. means of exchange.

In view of the fact that bitcoin investment and transaction rates are rather low compared to other assets, there is no serious risk or, even, imminent threat to financial or economic 
stability. However, if global trends led to an increased use of bitcoin or other virtual currencies, values of major fiat currencies and global monetary policy would be seriously affected. Considering the global decentralized character of bitcoin and its independence from any central bank or supranational body, any regulatory supervision may be difficult to exercise (Baur, Hong and Lee, 2018).

Bitcoin can be used either for payments, or as an investment. On a long-term basis, we must not panic seeing it drop, as this virtual currency is good for future appreciation.

What is the liquidity of bitcoin?

Using high-rate data, we found that a specific number of tweets positively influences bitcoin liquidity. The presented study measures the impact of tweets on the liquidity on a timeline - the tweets witness a favourable effect within five to ten minutes with a duration of approximately one hour. Our empirical evidence suggests that investors' assiduous attention may significantly improve bitcoin liquidity in the real time, which increases the effect of prices on the bitcoin market (Choi, 2021). Research carried out by Scharnowski (2021) contributes to understanding bitcoin liquidity, which has recently seen dramatic progress. The liquidity shifts between individual stock markets, so that academic workers and experts can choose from various places. The key factors behind the changes in the liquidity and trading activities mostly come from the bitcoin network, whereas the financial state of the US economy has only a negligible impact on bitcoin liquidity. The weekly liquidity patterns confirm the previous results, indicating activities of different groups of investors.

Bitcoin liquidity has recently marked a tremendous improvement. As contrasted to fiat currencies, bitcoin does not currently have centralized supply chain management and thus can be purchased for its market price.

\section{Conclusion}

The submitted article focused on answering the formulated hypotheses. Applying metaanalyses of available documents, we explored the position and role of bitcoin on the market. The objective was thereby fulfilled.

As one of the decisive factors of the Fourth Industrial Revolution, bitcoin closely interacts with the global economy (e. g. the International Energy Market), which provides valuable information for the further development of cryptocurrency markets. A social transformation has recently been accelerated dramatically owing to the considerable progress in the blockchain, internet of things, cloud computing, big data, artificial intelligence and other technologies. Among these modern conveniences, cryptocurrencies have great potential for supporting the reduction of international transaction fees and liquidity costs to successfully complete the flow of international wealth. Secondly, the continuing improvement in encrypting technologies and tightened supervision of the market curtails violent fluctuations in cryptocurrency prices, making them more appealing to the public. In this way, people rely more on bitcoin as a hedging asset. Thirdly, bitcoin is the first open platform for exchanging values on an internet scale. The blockchain technology supporting a large number of exchanges of values will inevitably cause a great boom in negotiable assets, generating a mountainous industrial wave which, in connection with other innovative forces, may accelerate social progress. The results show that bitcoin is closely related to other financial assets, thus giving space for further research aimed at better understanding and modelling the interaction between bitcoin and other financial assets.

As the situation on financial and cryptocurrency markets is subject to a continual change, our results can be somehow "obsolete". It is thereby necessary to carry out up-to-date analyses with the view to the economic expectation from bitcoin. 


\section{References}

1. Baur, D. G., Hong, K., \& Lee, A. D. (2018). Bitcoin: Medium of exchange or speculative assets? Journal of International Financial Markets, Institutions and Money, 54, 177189.

2. Borri, N. (2019). Conditional tail-risk in cryptocurrency markets. Journal of Empirical Finance, 50, 1 - 19.

3. Choi, H. (2021). Investor attention and bitcoin liquidity: Evidence from bitcoin tweets. Finance Research Letters, 39, 101555.

4. CoinMarketCap.com. (2021). Today's Cryptocurrency Prices by Market Cap [online]. Available of: https://coinmarketcap.com/ [cit. 2021-4-30]

5. Conlin, T., \& McGee, R. (2020). Safe haven or risky hazard? Bitcoin during the Covid19 bear market. Financial Research Letters, 35, 101607.

6. Dyhrberg, A. H. (2016). Hedging capabilities of bitcoin. Is it the virtual gold? Finance Research Letters, 16, 139-144.

7. Faghih Mohammadi Jalali, M., \& Heidari, H. (2020). Predicting changes in Bitcoin price using grey system theory. Financial Innovation, 6(1).

8. Figa-Talamanca, G., Focardi, S., \& Patacca, M. (2021). Common dynamic factors for cryptocurrencies and multiple pair-trading statistical arbitrages. Decisions in Economics and Finance.

9. Giudici, P., \& Abu-Hashish, I. (2019). What determines bitcoin exchange prices? A network VAR approach. Finance Research Letters, 28, 309-318.

10. Grobys, K. (2021). When the blockchain does not block: on hackings and uncertainty in the cryptocurrency market. Quantitative Finance, 21(8), 1267-1279.

11. Hazlett, P. K., \& Luther, W. J. (2020). Is bitcoin money? And what that means. The Quarterly Review of Economics and Finance, 77, 144-149.

12. Houben R, \& Snyers A. (2020). Crypto-assets - key developers, regula concerns and responses. [online]. Available of: https://www.europarl.europa.eu/RegData/etudes/STUD/2020/648779/IPOL_STU(2020 )648779_EN.pdf [cit. 2021-4-30]

13. Hudson, R., \& Urquhart, A. (2021). Technical trading and cryptocurrencies. Annals of Operations Research, 297(1-2), 191-220.

14. Kliestik, T., Valaskova, K., Lazaroiu, G., Kovacova, M., \& Vrbka, J. (2020). Remaining financially healthy and competitive: The role of financial predictors. Journal of Competitiveness, 12(1), 74-92.

15. Liu, X. F., Jiang, X., Liu, S., \& Tse, CH. K. (2021). Knowledge Discovery in Cryptocurrency knowledge discovery in cryptocurrency transactions: A survey. IEEE Access, 9, 37229-37254.

16. Lopez-Cabarcos, M. A., Perez-Pico, A. M., Piñeiro-CHousa, J., \& Sevic, A. (2021). Bitcoin volatility, stock market and investor sentiment. Are they connected? Finance Research Letters, 38, 101399.

17. Lucey, B. M., Vigne, S. A., Ballester, L., Barbopoulos, L., Brzeszczynski, J., Carchano, O., Dimic, N., Fernandez, V., Gogolin, F., Gonzalez-Urteaga, A., Goodell, J. W., Helbing, P., Ichev, R., Kearney, F., Laing, E., Larkin, C. J., Lindblad, A., Loncarski, I., Ly, K. C., Marinc, M., Mcgee, R. J., McGroarty, F., Neville, C., O'Hagan-Luff, M., Piljak, V., Sevic, A., Sheng, X., Stafylas, D., Urquhart, A., Versteeg, R., Vu, A. N., Wolfe, S., Yarovaya, L., \& Zaghini, A. (2018). Future directions in international 
financial integration research - A crowdsourced perspective. International Review of Financial Analysis, 55, 35-49.

18. Scharnowski, S. (2021). Understanding Bitcoin liquidity. Finance Research Letters, 38, 101477.

19. Shen, D., Urquhart, A., \& Wang, P. (2019). Does twitter predict Bitcoin? Economics Letters, 174, 118-122.

20. Stix, H. (2021). Ownership and purchase intention of crypto-assets: Survey results. Empirica, 48, 65-99.

21. Urquhart, A. (2016). The inefficiency of Bitcoin. Economics Letters, 148, 80-82.

22. Urquhart, A. (2018). What causes the attention of Bitcoin? Economics Letters, 166, 40-

23. Urquhart, A., \& Zhang, H. (2019). Is Bitcoin a hedge or safe haven for currencies? An intraday analysis. International Review of Financial Analysis, 63, 49-57.

24. Zhu, P., Zhang, X., Wu, Y., Zheng, H., Zhang, Y., \& Trinidad Segovia, J. E. (2021). Investor attention and cryptocurrency: Evidence from the Bitcoin market. PLoS ONE, 16(2), e0246331. 